\title{
A review of Adverse Effects of Targeted Temperature Management for Post-Cardiac Arrest Patients
}

\author{
Steven Wright ${ }^{1}$, Samantha Peralta ${ }^{1}$ and Leonard Ranasinghe ${ }^{2 *}$ \\ ${ }^{1}$ College of Medicine, California Northstate University, USA \\ ${ }^{2}$ Professor of Medical Education and Emergency Medicine, California Northstate University, USA
}

Contributed Equally: Steven Wright, Samantha Peralta and Leonard Ranasinghe

*Corresponding author: Leonard Ranasinghe, Professor of Medical Education and Emergency Medicine, College of Medicine, California Northstate University, Elk Grove, CA, USA

To Cite This Article: Steven W, Samantha P, Leonard R. A review of Adverse Effects of Targeted Temperature Management for Post-Cardiac Arrest Patients. 2020 - 9(1). AJBSR.MS.ID.001349. DOI: 10.34297/AJBSR.2020.09.001349.

Received: 㘹 May 21, 2020; Published: 畊 May 29, 2020

\begin{abstract}
Targeted Temperature Management has been the standard of care in post cardiac arrest since 2005. Despite significant advancements since its infancy, and a multitude of studies, discrepancies still exist as to its efficacy. By understanding the associated adverse effects, we can begin to delineate incongruencies to guide future research in order to progress towards a more uniform "high-quality" Targeted Temperature Management. Both anticonvulsant and antiarrhythmic therapy may provide an avenue towards improving Targeted Temperature Management
\end{abstract}

Keywords: Targeted Temperature Management, Adverse effects, Seizures, Myoclonus, Sepsis, Pneumonia, Malignant arrhythmias, Hypokalemia

Abbreviations: TH: Therapeutic Hypothermia, TTM: Targeted Temperature Management, ROSC: Return of Spontaneous Circulation, AE: Adverse Effects, VEB: Ventricular ectopic beats; QTc: corrected QT, MA: Malignant Arrhythmia.

\section{Review}

Therapeutic hypothermia (TH) has been the standard of care in post-cardiac arrest since 2005 following the recommendation of the International Liaison Committee on Resuscitation. The ILCOR's 2005 report recommended hypothermia induction for a duration of 12-24 hours in patients with return ofspontaneous circulation (ROSC) after ventricular fibrillation [1]. This recommendation followed data supported by two randomized controlled trials that demonstrated improvement in survival and neurologic outcome in patients who remained comatose following cardiac arrest when therapeutic hypothermia was performed $[2,3]$. Current guidelines by the American Heart Association continue to recommend the use of TH (now referred to as targeted temperature management (TTM)) [4]. Substantial progress has been made when looking at the multitude of scientific literature available. In its infancy, TTM protocol was severely lacking, providing a recommendation without a methodology as to how to best provide TTM. This data was largely unavailable at the time. Current recommendations now provide tighter regulations in regard to duration of TTM and when to initiate TTM following cardiac arrest as well as a recommendation against rapid infusion of cold IV fluids [4]. Although significant advancement has been made in TTM protocol, there remains a lack of consensus as to how to maximize its beneficial effects.

Throughout its existence contradictory results about the benefits of TTM have been published. In 2018, Kalra R, et al. argued that TTM may not actually improve neurologic outcome; considering the adverse effects of hypothermia on the body, it was argued that the risk may not outweigh the proposed benefits [5]. The large Hyperion study, however, confirmed the neurologic benefit of TTM following cardiac arrest [6]. Not only is there discrepancy in the efficacy of TTM, the assessed primary outcome (neurologic outcome vs survival) varies within the literature. Heterogeneity regarding assessment of primary outcomes, TTM duration, how TTM is administered, pharmacologic management during TTM, targeted temperatures and monitoring of TTM may all 
be confounders amongst the data producing contradictory results. With clear discrepancies, questions remain regarding the efficacy of TTM. Following the Hyperion trail, the literature began to advocate for the necessity of controlled, "high-quality" TTM in order to begin answering these questions. High quality TTM involves early initiation, pharmacologic management of shivering and facilitation of cooling, a specific target temperature in the cooling phase with a prolonged rewarming phase and avoidance of fever after TTM [7]. With increased homogeneity, it is argued that high quality TTM can maximize the proposed benefits by minimizing associated adverse outcomes. Perhaps the biggest debate amongst the available literature is whether or not TTM administration provides enough of a benefit to outweigh its many documented adverse effects (AE). AE of TTM have been documented within almost every organ system and will be discussed in this review. Advanced understanding of these AE is essential for maximizing the benefits of TTM and minimizing AE.

Seizure and myoclonus in the advanced critical care period (defined as days 1-7 after ROSC) are well known AE of TTM documented extensively in the literature [8-12]. Through both univariate and multivariate analysis, seizure and myoclonus have been strongly correlated with poor neurologic outcome. Anticonvulsant therapy has shown mixed effects on neurologic outcome with both improvement and detriment at hospital discharge [8]. However, anticonvulsant use could not be considered an independent variable in the analysis and provides opportunity for further investigation. The strongest predictors of poor neurologic outcome have been shown to be incomplete brainstem reflexes (including pupillary, oculocephalic, corneal), myoclonus, elevated neuron specific enolase concentration and nonreactive hypothermic EEG [13]. Not surprisingly, evidence of neurologic injury strongly correlates with a poor neurologic outcome. Further investigation is needed in understanding how to improve TTM administration in a way that greatly reduces the risk of neurologic injury. The 2015 report by the AHA addressed this need by identifying gaps in current knowledge and suggesting areas for further research. Amongst the most pertinent to this discussion is identifying if "the use of targeted temperature management, including various temperature targets, affects longterm neurocognitive and functional outcomes"[4].

Possibly one of the greatest adverse effects seen post cardiac arrest and through TTM administration is the damaging immunologic response. Widespread activation of the immunologic cascade increases the risk of infection following reperfusion after cardiac arrest [14]. Though TTM is standard of care, it has also been shown to increase the risk of infection [15]. Pneumonia and sepsis are prominent infectious etiologies associated with TTM. However, when assessing a primary outcome of neurologic outcome at hospital discharge, only sepsis demonstrated a strong association with poor neurologic outcome through a multivariate analysis [8].
Though pneumonia was not associated with a poor neurologic outcome, minimizing it post TTM is important for improvement in general patient outcome. Prophylactic antibiotic treatment to minimize pneumonia risk has been a suggested mechanism towards improving patient functional outcome. Currently, the literature fails to support a significant association between antibiotic therapy and improvement in patient mortality or neurologic outcome $[8,16]$. Although prophylactic antibiotic therapy may lower the incidence of pneumonia [8], further investigation is recommended before making conclusions about their effect on functional outcome.

Hemorrhage and thrombotic changes are established complications of non-medically induced hypothermia [17]. Thromboelastographic analysis of hypothermic patients has shown changes in multiple steps of the coagulation cascade, as well as delayed clot lysis [17]. Not surprisingly, similar complications have been reported in association with TTM, which is thought to be a result of a multitude of factors including elevated catecholamines, acidosis, tissue damage, and hypothermia-associated coagulopathy $[17,18]$. A study of 975 patients revealed increased risk of bleeding complications when patients underwent PCI angiography before TTM [18], while a sub-study of the TTM trial evaluated the effect of temperature on bleeding and coagulation showed no difference in risk between $33^{\circ} \mathrm{C}$ and $36^{\circ} \mathrm{C}$ [17]. Despite this evidence, only $4-5 \%$ of patients undergoing TTM were shown to have this complication $[8,18]$. Given the established neurologic benefit, the risk of these events is acceptable. Further work to delineate patient subsets who are at increased risk of serious hemorrhagic or thrombotic complications may help guide future clinical use of TTM.

EKG changes are also frequently observed in patients undergoing TTM. The presence of bradycardia during TTM is a potential predictor of positive outcome and was associated with an $18 \%$ improvement in mortality and a lower odds ratio of poor neurologic outcomes in a randomized trial [19]. Ventricular ectopic beats (VEB) were observed in the majority of patients undergoing TTM regardless of target temperature in another randomized trial [20]. The prevalence of VEB decreased as TTM progressed, and patients treated at $36^{\circ} \mathrm{C}$ had less VEB at the later time points, which continued to be significant after adjusting for possible confounders [20]. Despite this, the rate of ventricular tachycardia or fibrillation did not differ between groups, and the mortality and neurological outcome did not differ [20]. Similarly, the presence of Osborn waves increased with lower target temperature without increased ventricular arrhythmias [21]. Multiple studies have shown prolonged QT and corrected QT (QTc) while undergoing TTM, particularly in females and patients with anoxic brain injuries $[22,23]$. No difference was observed in the QT interval between patients experiencing malignant arrhythmia (MA) and those who did not, regardless of the use of known QT prolongating drugs and other variables such as electrolyte imbalances [22]. 
MA (defined as ventricular fibrillation, sustained ventricular tachycardia, torsades de pointes) is a dangerous complication of cardiac arrest, regardless of use of TTM, and is associated with significant mortality for post cardiac arrest patients [24,25]. Similar to the changes observed in VEB prevalence, the number of defibrillations was significantly higher in patients who developed MA [24]. Additionally, patients with MA had longer time to ROSC, which has also been shown as a predictor of outcome $[24,26]$. The rate of MA did not vary with target temperature but was correlated with more aggressive decrease in body temperature in the first 6 hours of treatment [24]. This follows other studies which have suggested rate of temperature decline, and thus strength of heat homeostasis, as a possible predictor of mortality and outcome $[24,26]$. The use of vasopressors was associated with post resuscitation ventricular arrhythmias, potentially showing an avenue to identify high-risk patients [23].

Electrolyte changes, which are known to predispose patients to arrhythmias outside of TTM, are common during TTM and have been proposed as a possible causative factor of arrhythmias, though the data on this remains unclear. Abnormal potassium levels are common during TTM, with both hypokalemia and hyperkalemia being reported during all stages of TTM (86\% and $32 \%$ respectively) [27]. There does not appear to be an association between hypokalemia and ventricular arrhythmias. A trend between hypokalemia and MA was noted in one study, though not statistically significant [24], and multivariable regression showed no association between hypokalemia or hyperkalemia and recurrent ventricular arrhythmias or poor neurologic outcomes [27]. Interestingly, hypokalemia was associated with decreased odds of death prior to hospital discharge in one study, potentially pointing toward hypokalemia as a protective mechanism by the body [27]. The rate of decline in potassium levels in the first 12 hours of treatment is linked to an increased rate of MA, consistent with the effects of temperature decline rate, which may serve as a potential predictive factor [27]. Glycemic changes are also commonly observed during TTM. Hyperglycemia was observed in $45.6 \%$ of patients, though no significant impacts on neurologic outcome were observed as a result [8]. Hypoglycemia, on the other hand, was associated with poor neurologic outcome and was observed in $23 \%$ of patients indicating the need for aggressive management to avoid hypoglycaemia [8].

Controlling for the multitude of adverse effects seen in TTM administration remains a significant challenge. Attempts to control for and isolate these effects through investigation is difficult, as many AE are multifactorial in causality. For example, neurologic injury has shown to be a predictive factor of poor neurologic outcome[24]. In the referenced literature, anticonvulsants were administered following the incidence of seizure (a known cause of neurologic injury), but the benefits of their use is unclear due to the inability to isolate anticonvulsant use and seizure [8]. Delineation of the effects of anticonvulsants on neurologic outcome is necessary before making further conclusions towards their effectiveness. Nonetheless, anticonvulsants may provide a potential avenue for further investigation. Given the association between increased rate of decline in serum potassium levels and MA [27], investigation into the use of prophylactic antiarrhythmics in the subset of patients with rapid decline may prove insightful. Many of these associated risks are acceptable considering the substantial benefit TTM has been shown to provide. Secondary to the fact that the origins of the $\mathrm{AE}$ are multifactorial, it is important to further investigate where in the pathway TTM is contributing to, and/or causing these AE.Identifying the underlying mechanisms resulting in poor functional outcome and poor neurologic outcome poses the greatest potential for the future of TTM. By understanding/ isolating the underlying mechanisms of the associated $\mathrm{AE}$, further recommendations for TTM can be made. Arguably, the future of TTM resides in the ability to administer a "high quality" TTM with early initiation, well-controlled pharmacologic management, a specific target temperature in the cooling phase with a prolonged rewarming phase and avoidance of fever after TTM [7]. Through further investigation of the adverse effects of TTM, we can begin to tighten the parameters of TTM to maximize its benefit to patients.

\section{References}

1. (2005) Part 4: Advanced life support. Resuscitation 67(2): 213-247.

2. The Hypothermia after Cardiac Arrest Study Group (2002)Mild Therapeutic Hypothermia to Improve the Neurologic Outcome after Cardiac Arrest. New England Journal of Medicine 346(8): 549-556.

3. Bernard SA, Timothy W Gray, Michael D Buist, Bruce M Jones, William Silvester, et al. (2002) Treatment of Comatose Survivors of Out-ofHospital Cardiac Arrest with Induced Hypothermia. New England Journal of Medicine 346(8): p. 557-563.

4. Donnino Michael W, Lars W Andersen, Katherine M Berg, Joshua C Reynolds, Jerry P Nolan, et al. (2015) Temperature Management After Cardiac Arrest. Circulation 132(25): 2448-2456.

5. Rajat Kalra, Garima Arora, Nirav Patel, Rajkumar Doshi, Lorenzo Berra, et al. (2018) Targeted Temperature Management After Cardiac Arrest: Systematic Review and Meta-analyses. Anesthesia and analgesia126(3): 867-875.

6. Jean-Baptiste Lascarrou, Hamid Merdji, Amélie Le Gouge, Gwenhael Colin, Guillaume Grillet, et al. (2019) Targeted Temperature Management for Cardiac Arrest with Nonshockable Rhythm. New England Journal of Medicine 381(24): 2327-2337.

7. Taccone FS, E Picetti, JL Vincent (2020) High Quality Targeted Temperature Management (TTM) After Cardiac Arrest. Critical Care 24(1): p. 6.

8. Young-Min Kim, Chun Song Youn, Soo Hyun Kim, Byung Kook Lee, In Soo Cho, et al. (2015) Adverse events associated with poor neurological outcome during targeted temperature management and advanced critical care after out-of-hospital cardiac arrest. Critical care 19(1): p. 283-283.

9. Niklas Nielsen, JørnWetterslev, Tobias Cronberg, David Erlinge, Yvan Gasche, et al. (2013) Targeted temperature management at 33 degrees C versus 36 degrees C after cardiac arrest. N Engl J Med 369(23): 21972206. 
10. Nielsen N (2011) Adverse events and their relation to mortality in out-ofhospital cardiac arrest patients treated with therapeutic hypothermia. Crit Care Med 39(1): 57-64.

11. Krumholz A, BJ Stern, HD Weiss (1988) Outcome from coma after cardiopulmonary resuscitation: relation to seizures and myoclonus. Neurology 38(3): 401-405.

12. Ram Mani, Sarah E Schmitt, Maryann Mazer, Mary E Putt, David F Gaieski, et al. (2012) The frequency and timing of epileptiform activity on continuous electroencephalogram in comatose post-cardiac arrest syndrome patients treated with therapeutic hypothermia. Resuscitation 83(7): 840-847.

13. Oddo M, AO Rossetti (2014) Early multimodal outcome prediction after cardiac arrest in patients treated with hypothermia. Crit Care Med 42(6): 1340-1347.

14. Neumar Robert W,Jerry P Nolan, Christophe Adrie, MayukiAibiki, Robert A Berg, et al. (2008) Post-Cardiac Arrest Syndrome. Circulation118(23): 2452-2483.

15. Mongardon N, Perbet S, Lemiale V, Dumas F, Poupet H, et al. (2011) Infectious complications in out-of-hospital cardiac arrest patients in the therapeutic hypothermia era. Critical Care Medicine 39(6).

16. Takashi Tagami, Hiroki Matsui, Masamune Kuno, Yuuta Moroe, Junya Kaneko T, et al. (2016) Early antibiotics administration during targeted temperature management after out-of-hospital cardiac arrest: a nationwide database study. BMC Anesthesiology 16(1): 89.

17. Marrit Jacob, Christian Hassager, John Bro-Jeppesen, Sisse R Ostrowski, JaobHartvig, et al. (2015) The effect of targeted temperature management on coagulation parameters and bleeding events after outof-hospital cardiac arrest of presumed cardiac cause. Resuscitation96: p. 260-267.

18. Nielsen N (2009) Outcome, timing and adverse events in therapeutic hypothermia after out-of-hospital cardiac arrest. Acta Anaesthesiol Scand 53(7): 926-934

19. Thomsen JH, Nielsen N, Hassager C, Wanscher M, Pehrson S, et al.(2016) Bradycardia During Targeted Temperature Management: An Early
Marker of Lower Mortality and Favorable Neurologic Outcome in Comatose Out-of-Hospital Cardiac Arrest Patients. Crit Care Med 44(2): 308-318.

20. Jakob Hartvig Thomsen, Jesper Kjaergaard, Claus Graff, Steen Pehrson, David Erlinge, et al. (2016) Ventricular ectopic burden in comatose survivors of out-of-hospital cardiac arrest treated with targeted temperature management at $33^{\circ} \mathrm{C}$ and $36^{\circ} \mathrm{C}$. Resuscitation 102: 98-104.

21. Edina Hadziselimovic, Jakob Hartvig Thomsen, Jesper Kjaergaard, Lars Køber, Claus Graff, et al. (2018) Osborn waves following out-of-hospital cardiac arrest-Effect of level of temperature management and risk of arrhythmia and death. Resuscitation 128: 119-125.

22. Zachary Rosol, David F Miranda, Yader Sandoval, Bradley A Bart, Stephen W Smith, et al. (2017) The effect of targeted temperature management on QT and corrected QT intervals in patients with cardiac arrest. J Crit Care 39: 182-184.

23. Piktel JS, LD Wilson (2018) Targeted temperature management after sudden cardiac arrest: Proarrhythmic or antiarrhythmic? Probably both. J Crit Care 46: 149-150.

24. Christoph Adler, Felix Schregel, Tobias Heller, Martin Hellmich, Joana Adler, et al. (2019) Malignant Arrhythmias During Induction of Target Temperature Management After Cardiac Arrest. Therapeutic Hypothermia and Temperature Management.

25. Bigger JT (1983) Definition of benign versus malignant ventricular arrhythmias: targets for treatment. Am J Cardiol 52(6): p. 47c-54c.

26. Steve Lin, Damon C Scales, Paul Dorian, Alexander Kiss, Matthew R Common, et al. (2014) Targeted temperature management processes and outcomes after out-of-hospital cardiac arrest: an observational cohort study. Crit Care Med 42(12): 2565-2574.

27. ArashNayeri, Hannah Gluck, Eric Farber-Eger, Srikanth Krishnan, Kamran Shamsa, et al. (2017) Temporal Pattern and Prognostic Significance of Hypokalemia in Patients Undergoing Targeted Temperature Management Following Cardiac Arrest. Am J Cardiol 120(7): 1110-1113. 